\title{
A description based on Schubert classes of cohomology of flag manifolds
}

by

\author{
Masaki Nakagawa (Takamatsu)
}

\begin{abstract}
We describe the integral cohomology rings of the flag manifolds of types $B_{n}, D_{n}, G_{2}$ and $F_{4}$ in terms of their Schubert classes. The main tool is the divided difference operators of Bernstein-Gelfand-Gelfand and Demazure. As an application, we compute the Chow rings of the corresponding complex algebraic groups, recovering thereby the results of R. Marlin.
\end{abstract}

1. Introduction. Let $K$ be a compact connected Lie group and $T$ its maximal torus. The homogeneous space $K / T$, called the flag manifold, plays an important role in algebraic topology, algebraic geometry and representation theory.

In this paper, we are concerned with the integral cohomology of the flag manifold $K / T$. As is well known, there are two descriptions of this cohomology. The first one is the "Borel presentation", due to A. Borel [2], which identifies the rational cohomology ring of $K / T$ with the quotient ring of a polynomial ring by its ideal generated by $W$-invariants of positive degrees, where $W$ is the Weyl group of $K$. Combining Borel's result and the known structures of the $\bmod p$ cohomology rings of $K, \mathrm{H}$. Toda gave general descriptions of the integral cohomology rings of $K / T$ for all $K$ simple [18]. So far the integral cohomology rings of flag manifolds for all compact simply connected simple Lie groups are determined (see [2], [3], [19], [14], [15]). The second is the "Schubert presentation" which describes the integral cohomology $H^{*}(K / T ; \mathbb{Z})$ in terms of the Schubert classes corresponding to the Schubert varieties derived from the Bruhat decomposition of $G=K^{\mathbb{C}}$, the complexification of $K$.

In the Borel presentation, which is given by generators and relations, the ring structure of $H^{*}(K / T ; \mathbb{Z})$ can be relatively easy to obtain. How-

2000 Mathematics Subject Classification: Primary 57T15; Secondary 14M15.

Key words and phrases: flag manifolds, Schubert calculus, Chow rings.

Partially supported by the Grant-in-Aid for Scientific Research (C) 18540106, Japan Society for the Promotion of Science. 
ever, the generators in this presentation have little geometric meaning. In contrast, in the Schubert presentation, the Schubert classes correspond to the geometric objects - the Schubert varieties - and they form an additive basis for $H^{*}(K / T ; \mathbb{Z})([5])$. As a disadvantage, the multiplicative structure among them is closely related to the intersection multiplicities, and is highly complicated in general.

Up to now, there have been several attempts to establish a connection between the two descriptions for some types of spaces (see, e.g., [1], [8], [11]). The main aim of this paper also falls in this category. More precisely, we express the ring generators in the Borel presentation of $H^{*}(K / T ; \mathbb{Z})$ for $K=S O(n), G_{2}$ and $F_{4}$ in terms of Schubert classes. For this, we make use of the "divided difference operators" introduced independently by BernsteinGelfand-Gelfand [1] and Demazure [6]. For $K$ as above, there exist extra generators of degrees greater than two. So we cannot apply the divided difference operators directly to these higher generators. Fortunately, using the classical fact that, rationally, the cohomology of $K / T$ is generated as a ring by two-dimensional elements, and the integral cohomology of $K / T$ has no torsion, we can carry out the computation. An additional aim of this paper is to apply our results to recovering the Chow rings of the complex algebraic groups $\mathrm{SO}(n)$, $\operatorname{Spin}(n), \mathrm{G}_{2}$ and $\mathrm{F}_{4}$, which were originally computed by R. Marlin [13]. (In this paper, we denote the compact Lie groups, e.g., by $S O(n), \operatorname{Spin}(n), G_{2}, F_{4}$, while their complexifications by $\mathrm{SO}(n), \operatorname{Spin}(n), \mathrm{G}_{2}$, $\mathrm{F}_{4}$ respectively.) In order to determine the Chow rings of the corresponding flag manifolds, Marlin relied on the result of Demazure [6] which describes them as the "cohomology rings of the root system", and he made elaborate computations. In this paper, we simplify Marlin's computations, using the Borel presentation of $H^{*}(K / T ; \mathbb{Z})$ and our result mentioned above.

The paper is organized as follows. In Section 2, we briefly review the cohomology of flag manifolds, emphasizing the difference between the Borel and Schubert presentations. In Section 3, we introduce the divided difference operators of Bernstein-Gelfand-Gelfand and Demazure and collect the results used later. Sections 4 to 6 are devoted to computations, and we obtain there the main results of this paper (see Propositions 4.3, 4.7, 5.3 and 6.5). In Section 7, following Grothendieck's remark $\left(\left[9\right.\right.$, p. 21 , Remarques $\left.\left.2^{\circ}\right]\right)$, we compute the Chow rings of $\mathrm{SO}(n), \operatorname{Spin}(n), \mathrm{G}_{2}$ and $\mathrm{F}_{4}$ (see Theorems 7.2, 7.3, 7.4 and 7.1).

We observe that the method of this paper can also be applied to the exceptional Lie groups $E_{6}, E_{7}$ and $E_{8}$. Indeed, we succeeded in computing the Chow rings of the complex algebraic groups $\mathrm{E}_{6}$ and $\mathrm{E}_{7}$ in [12].

Acknowledgments. Firstly, we thank Július Korbaš for reading carefully the manuscript and giving us useful comments and William Fulton for 
kindly pointing out an error concerning the Schubert classes in the earlier version of the manuscript. Secondly, we thank Mamoru Mimura for giving us various suggestions. Finally, we thank Shizuo Kaji for the program using Maple, which confirmed our computations.

2. The cohomology of flag manifolds. In this section we briefly review the Borel and Schubert presentations of the cohomology of flag manifolds.

We introduce the notation that is needed in the following.

- $K$ : a compact simply connected simple Lie group of rank $l$;

- $T$ : a maximal torus of $K$;

- $G=K^{\mathbb{C}}$ : a complexification of $K$;

- $B$ : a Borel subgroup containing $T$;

- $\mathfrak{t}$ : the Lie algebra of $T ; \mathfrak{t}^{*}$ : the dual space of $\mathfrak{t}$;

- $(\cdot \mid \cdot)$ : the invariant inner product on $\mathfrak{t}\left(\right.$ or on $\left.\mathfrak{t}^{*}\right)$;

- $\Delta \subset \mathfrak{t}^{*}$ : the root system with respect to $T$;

- $\Delta^{+}$: the set of positive roots, $\Delta^{-}=-\Delta^{+}$;

- $\Pi=\left\{\alpha_{1}, \ldots, \alpha_{l}\right\}$ : the system of simple roots;

- $\alpha^{\vee}=2 \alpha /(\alpha \mid \alpha)$ : the coroot corresponding to $\alpha \in \Delta$;

- $\omega_{i}(1 \leq i \leq l)$ : the $i$ th fundamental weight, satisfying $\left(\omega_{i} \mid \alpha_{j}^{\vee}\right)=\delta_{i j}$;

- $s_{i}=s_{\alpha_{i}}(1 \leq i \leq l)$ : the reflection corresponding to the simple root $\alpha_{i}$;

- $W=W(K)$ : the Weyl group of $K$ generated by the simple reflections $S=\left\{s_{1}, \ldots, s_{l}\right\}$

- $l(w)$ : the length of an element $w \in W$ with respect to $\left\{s_{1}, \ldots, s_{l}\right\}$;

- $w_{0}$ : the longest element of $W$;

- $e_{i}\left(x_{1}, \ldots, x_{n}\right)$ : the $i$ th elementary symmetric function in variables $x_{1}, \ldots, x_{n}$.

Now we review the Borel presentation. The inclusion $T \hookrightarrow K$ induces the classical fibration

$$
K / T \stackrel{\iota}{\rightarrow} B T \stackrel{\varrho}{\rightarrow} B K,
$$

where $B T$ (resp. $B K$ ) denotes the classifying space of $T$ (resp. $K$ ). The induced homomorphism

$$
c=\iota^{*}: H^{*}(B T ; \mathbb{Z}) \rightarrow H^{*}(K / T ; \mathbb{Z})
$$

is called the characteristic homomorphism and plays a crucial role in Borel's work. The Weyl group $W$ acts naturally on $T$, hence on $H^{2}(B T ; \mathbb{Z})$. We extend this natural action of $W$ to the whole $H^{*}(B T ; \mathbb{Z})$ and also to $H^{*}(B T ; \mathbb{F})$ $=H^{*}(B T ; \mathbb{Z}) \otimes_{\mathbb{Z}} \mathbb{F}$, where $\mathbb{F}$ is any field. We denote by $H^{*}(B T ; \mathbb{Z})^{W}$ (resp. $\left.H^{*}(B T ; \mathbb{F})^{W}\right)$ the ring of $W$-invariants in $H^{*}(B T ; \mathbb{Z})\left(\operatorname{resp} . H^{*}(B T ; \mathbb{F})\right.$ ). Then one of the main results of Borel can be stated as follows. 
Theorem 2.1 (Borel [2]). Let $\mathbb{F}$ be a field of characteristic zero. Then the characteristic homomorphism induces an isomorphism

$$
\bar{c}: H^{*}(B T ; \mathbb{F}) /\left(H^{+}(B T ; \mathbb{F})^{W}\right) \rightarrow H^{*}(K / T ; \mathbb{F}),
$$

where $\left(H^{+}(B T ; \mathbb{F})^{W}\right)$ is the ideal in $H^{*}(B T ; \mathbb{F})$ generated by the $W$-invariants of positive degrees.

In particular, one can reduce the computation of the rational cohomology ring $H^{*}(K / T ; \mathbb{Q})$ to that of the ring of invariants $H^{*}(B T ; \mathbb{Q})^{W}$. Observe that $H^{*}(K / T ; \mathbb{Q})$ is generated by $H^{2}(K / T ; \mathbb{Q})$ as a ring. In order to determine the integral cohomology ring $H^{*}(K / T ; \mathbb{Z})$, we need further considerations. In [18], Toda established a method to describe the integral cohomology ring $H^{*}(K / T ; \mathbb{Z})$ by a minimal system of generators and relations, from the $\bmod p$ cohomology rings $H^{*}(K ; \mathbb{Z} / p \mathbb{Z})$ and the rational cohomology ring $H^{*}(K / T ; \mathbb{Q})$. In general, besides the two-dimensional generators, there are extra generators of higher degrees, and hence the characteristic homomorphism $c$ is not surjective over $\mathbb{Z}$ in that case. Along the lines of Toda's method, the integral cohomology rings of flag manifolds for all compact simply connected simple Lie groups have been computed (see [2], [3], [19], [14], [15]). However, as mentioned in the introduction, the generators have less geometric meaning in the Borel presentation.

We pass to reviewing the Schubert presentation. Recall the Bruhat decomposition,

$$
G=\coprod_{w \in W} B \dot{w} B
$$

where $\dot{w}$ denotes any representative of $w$ in $W=N_{K}(T) / T ; N_{K}(T)$ is the normalizer of $T$ in $K$. It induces a cell decomposition,

$$
G / B=\coprod_{w \in W} B \dot{w} B / B
$$

where

$$
X_{w}^{\circ}=B \dot{w} B / B \cong \mathbb{C}^{l(w)}
$$

is called the $S$ chubert cell. Note that we have a homeomorphism $K / T \cong G / B$ by the Iwasawa decomposition. The Schubert variety $X_{w}$ is defined to be the closure of $X_{w}^{\circ}$. Then it is known that

$$
X_{w}=\coprod_{v \leq w} X_{v}^{\circ}
$$

where $\leq$ is the Bruhat-Chevalley ordering. The fundamental class $\left[X_{w}\right]$ of $X_{w}$ lies in $H_{2 l(w)}(G / B ; \mathbb{Z})$. We define the cohomology class $Z_{w} \in$ $H^{2 l(w)}(G / B ; \mathbb{Z})$ as the Poincaré dual of $\left[X_{w_{0} w}\right] \in H_{2 N-2 l(w)}(G / B ; \mathbb{Z})$, where $N$ is the complex dimension of the flag manifold $G / B$. We call $Z_{w}$ the Schubert class. The Schubert classes $\left\{Z_{w}\right\}_{w \in W}$ form an additive basis for 
$H^{*}(G / B ; \mathbb{Z})$. We refer to $\left\{Z_{w}\right\}_{w \in W}$ as the Schubert basis. In order to complete the description of $H^{*}(G / B ; \mathbb{Z})$, we have to compute the intersection multiplicities. Namely, given $u, v \in W$, we can put

$$
Z_{u} \cdot Z_{v}=\sum_{\substack{w \in W \\ l(u)+l(v)=l(w)}} a_{u, v}^{w} Z_{w}
$$

for some integers $a_{u, v}^{w}$, and we have to determine these "structure constants" $a_{u, v}^{w}$. For this problem, several results are available. For example, we have the following

Theorem 2.2 (Chevalley formula [5]). If $w \in W$ and $\alpha \in \Pi$, then

$$
Z_{s_{\alpha}} \cdot Z_{w}=\sum_{\substack{\beta \in \Delta^{+} \\ l\left(w s_{\beta}\right)=l(w)+1}}\left(\beta^{\vee} \mid \omega_{\alpha}\right) Z_{w s_{\beta}} .
$$

For recent developments in the Schubert calculus, in particular, on multiplying Schubert classes, see also [7], [16].

3. Schubert calculus on flag manifolds. As reviewed in the previous section, there are two different ways of describing the integral cohomology ring of $K / T$, and therefore we have two bases for $H^{*}(K / T ; \mathbb{Z})$. One is the "algebraic basis" derived from the Borel presentation and the other is the "geometric basis" $\left\{Z_{w}\right\}_{w \in W}$ consisting of the Schubert classes. It is interesting to know how these two bases are related. More precisely, we wish to express the ring generators obtained in the Borel presentation in terms of the Schubert basis. Our main tool will be the divided difference operators introduced independently by Bernstein-Gelfand-Gelfand [1] and Demazure [6]. We now recall their definition. For $\alpha \in \Delta$, we define an endomorphism of $H^{*}(B T ; \mathbb{Z})$ by

$$
\Delta_{\alpha}(u)=\frac{u-s_{\alpha}(u)}{\alpha}, \quad u \in H^{*}(B T ; \mathbb{Z}) .
$$

Definition 3.1. For $w \in W$, we define the operator

$$
\Delta_{w}=\Delta_{\alpha_{i_{1}}} \circ \cdots \circ \Delta_{\alpha_{i_{k}}}
$$

on $H^{*}(B T ; \mathbb{Z})$ lowering the degree by $2 l(w)$, where $w=s_{i_{1}} \cdots s_{i_{k}}$ is a reduced decomposition of $w$.

One can show that the operator $\Delta_{w}$ is well defined, i.e., it is independent of the choice of the reduced decomposition of $w$.

Note that the divided difference operators $\Delta_{\alpha}, \alpha \in \Delta$, are characterized by the following two properties:

$$
\begin{gathered}
\Delta_{\alpha}\left(\omega_{\beta}\right)=\delta_{\alpha \beta}, \\
\Delta_{\alpha}(u v)=\Delta_{\alpha}(u) v+s_{\alpha}(u) \Delta_{\alpha}(v)
\end{gathered}
$$

for $u, v \in H^{*}(B T ; \mathbb{Z})$. 
The characteristic homomorphism

$$
c: H^{*}(B T ; \mathbb{Z}) \rightarrow H^{*}(K / T ; \mathbb{Z})
$$

can be described by the divided difference operators. Since $\left\{Z_{w}\right\}_{w \in W}$ is a $\mathbb{Z}$-basis for $H^{*}(K / T ; \mathbb{Z})$, we can put

$$
c(f)=\sum_{l(w)=k} a_{w} Z_{w}, \quad a_{w} \in \mathbb{Z},
$$

for a polynomial $f \in H^{2 k}(B T ; \mathbb{Z})$. So we have to determine the coefficients $a_{w}$. This problem was solved independently by Bernstein-Gelfand-Gelfand [1] and Demazure [6].

Theorem 3.2 (Bernstein-Gelfand-Gelfand [1], Demazure [6]). For a polynomial $f \in H^{2 k}(B T ; \mathbb{Z})$, we have

$$
c(f)=\sum_{l(w)=k} \Delta_{w}(f) Z_{w} .
$$

In particular, for $\alpha \in \Pi$, we have

$$
c\left(\omega_{\alpha}\right)=Z_{s_{\alpha}} .
$$

In addition, using the divided difference operators, we can express an arbitrary Schubert class $Z_{w}$ as a polynomial in the variables $Z_{s_{i}}$. This is the Giambelli formula which we now recall (for details, see [10, Section 3]). Consider the element

$$
d=\prod_{\alpha \in \Delta^{+}} \alpha .
$$

Then we have

Theorem 3.3 (Giambelli formula). The Schubert class $Z_{w}$ corresponding to $w \in W$ is given by

$$
Z_{w}=c\left(\Delta_{w^{-1} w_{0}}\left(\frac{d}{|W|}\right)\right) .
$$

In Sections 4-6, we exploit the above theorems to find the correspondence between "algebraic bases" and "geometric bases" in the cases of $K=S O(n)$, $G_{2}$ and $F_{4}$.

4. The cases of $B_{n}$ and $D_{n}$. In this section, we consider the special orthogonal group $S O(n)$. First we deal with the odd special orthogonal group $S O(2 n+1)$. Let $T^{n}$ be the standard maximal torus of $S O(2 n+1)$, 


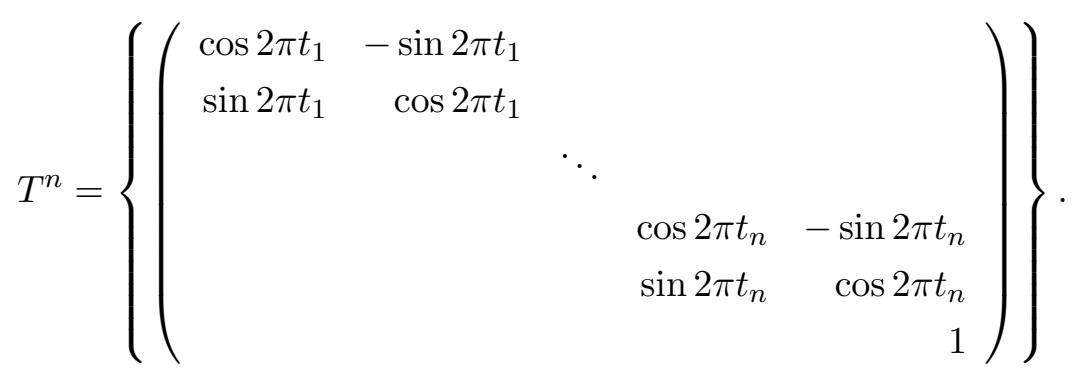

Then we have an isomorphism

$$
H^{*}\left(B T^{n} ; \mathbb{Z}\right)=\mathbb{Z}\left[t_{1}, \ldots, t_{n}\right] .
$$

The system of simple roots is

$$
\Pi=\left\{\alpha_{1}=t_{1}-t_{2}, \alpha_{2}=t_{2}-t_{3}, \ldots, \alpha_{n-1}=t_{n-1}-t_{n}, \alpha_{n}=t_{n}\right\} .
$$

The corresponding fundamental weights $\left\{\omega_{i}\right\}_{1 \leq i \leq n}$ are

$$
\begin{aligned}
\omega_{i} & =t_{1}+\cdots+t_{i} \quad(1 \leq i \leq n-1), \\
\omega_{n} & =\frac{1}{2}\left(t_{1}+\cdots+t_{n}\right) .
\end{aligned}
$$

Let $s_{i}(1 \leq i \leq n)$ be the reflection corresponding to $\alpha_{i}(1 \leq i \leq n)$. Then the Weyl group $W(S O(2 n+1))$ is finite and is generated by $s_{i}(1 \leq i \leq n)$ which act on $\left\{t_{i}\right\}_{1 \leq i \leq n}$ as permutations and sign changes:

$$
W(S O(2 n+1))=\left\langle s_{1}, \ldots, s_{n}\right\rangle \cong \mathcal{S}_{n} \ltimes(\mathbb{Z} / 2 \mathbb{Z})^{n},
$$

where $\mathcal{S}_{n}$ is the symmetric group of $n$ letters and $\ltimes$ means semidirect product.

Now we recall the Borel presentation of $H^{*}\left(S O(2 n+1) / T^{n} ; \mathbb{Z}\right)$, which was probably known, in some form, already to Borel. However, in an explicit form, it first appeared in [19] as far as the author knows.

Theorem 4.1 (Toda-Watanabe [19, Theorem 2.1]). The integral cohomology ring of $S O(2 n+1) / T^{n}$ is

$$
\begin{aligned}
H^{*}\left(S O(2 n+1) / T^{n} ; \mathbb{Z}\right)= & \mathbb{Z}\left[t_{1}, \ldots, t_{n}, \gamma_{1}, \ldots, \gamma_{n}\right] / \\
& \left(\begin{array}{l}
c_{i}-2 \gamma_{i}(1 \leq i \leq n), \\
\gamma_{2 k}+\sum_{i=1}^{2 k-1}(-1)^{i} \gamma_{i} \gamma_{2 k-i}(1 \leq k \leq n)
\end{array}\right),
\end{aligned}
$$

where we denote by the same symbols $t_{i} \in H^{2}\left(S O(2 n+1) / T^{n} ; \mathbb{Z}\right)$ the images of $t_{i} \in H^{2}\left(B T^{n} ; \mathbb{Z}\right)$ under the homomorphism $c, c_{i}=e_{i}\left(t_{1}, \ldots, t_{n}\right)(1 \leq i$ $\leq n)$, and $\gamma_{i}=0$ for $i>n$.

We wish to express the algebra generators $\left\{t_{1}, \ldots, t_{n}, \gamma_{1}, \ldots, \gamma_{n}\right\}$ in terms of Schubert classes. For simplicity, we denote the Schubert class corresponding to $w=s_{i_{1}} \cdots s_{i_{k}}$ by $Z_{i_{1} \cdots i_{k}}$, although the reduced decomposition of a 
Weyl group element may not be unique. The correspondence between elements of degree 2 is easy. By (4.1), we have

$$
\begin{aligned}
t_{1} & =\omega_{1}, \\
t_{i} & =-\omega_{i-1}+\omega_{i} \quad(2 \leq i \leq n-1), \\
t_{n} & =-\omega_{n-1}+2 \omega_{n} .
\end{aligned}
$$

Since $c\left(\omega_{i}\right)=Z_{i}$, it follows from (4.3) that

$$
\begin{aligned}
t_{1} & =Z_{1}, \\
t_{i} & =-Z_{i-1}+Z_{i} \quad(2 \leq i \leq n-1), \\
t_{n} & =-Z_{n-1}+2 Z_{n} .
\end{aligned}
$$

Next, for $\gamma_{k}(1 \leq k \leq n)$, we can put

$$
\gamma_{k}=\sum_{l(w)=k} a_{w} Z_{w}
$$

for some integers $a_{w}$. We need to determine the coefficients $a_{w}$. To this end, we make use of the divided difference operators recalled in the previous section. In this case, the characteristic homomorphism $c$ is not surjective over $\mathbb{Z}$ and $\gamma_{k}(1 \leq k \leq n)$ is not in the image of $c$. (Strictly speaking, we should consider the spinor group, because the special orthogonal group is not simply connected. In that case, $\gamma_{1}$ is in the image of $c$.) However, $2 \gamma_{k}=c_{k}$ is in the image of $c$. So we can apply Theorem 3.2 to the polynomial $c_{k}=e_{k}\left(t_{1}, \ldots, t_{n}\right)$. Thus

$$
c_{k}=\sum_{l(w)=k} \Delta_{w}\left(c_{k}\right) Z_{w} .
$$

Let us compute $\Delta_{w}\left(c_{k}\right)$, where $l(w)=k$ for fixed $k(1 \leq k \leq n)$. For convenience of computation, we introduce the notation

$$
c_{l}^{(m)}=e_{l}\left(t_{1}, \ldots, t_{m}\right) \quad(1 \leq m \leq n, 1 \leq l \leq m),
$$

so that $c_{k}=c_{k}^{(n)}$.

We need the following auxiliary result.

Lemma 4.2. For fixed $k, 1 \leq k \leq n$, we have

(1) $\Delta_{i}\left(c_{k}^{(n)}\right)=0(1 \leq i \leq n-1)$,

(2) $\Delta_{n}\left(c_{k}^{(n)}\right)=2 c_{k-1}^{(n-1)}$,

(3) $\Delta_{i}\left(c_{k-j}^{(n-j)}\right)=0(1 \leq i \leq n-j-1,1 \leq j \leq n-1)$,

(4) $\Delta_{n-j}\left(c_{k-j}^{(n-j)}\right)=c_{k-j-1}^{(n-j-1)}(1 \leq j \leq n-1)$. 
Proof. (1) and (3) follow from the definition of $\Delta_{i}$ and the fact that $c_{k}^{(n)}=e_{k}\left(t_{1}, \ldots, t_{n}\right)\left(\operatorname{resp} . c_{k-j}^{(n-j)}=e_{k-j}\left(t_{1}, \ldots, t_{n-j}\right)\right)$ is invariant under the action of $s_{i}(1 \leq i \leq n-1)$ (resp. $s_{i}(1 \leq i \leq n-j-1)$ ).

By (4.3), we have, for $1 \leq i \leq n-1$,

$$
\Delta_{i}\left(t_{j}\right)= \begin{cases}1 & (j=i), \\ -1 & (j=i+1), \\ 0 & (j \neq i, i+1)\end{cases}
$$

and

$$
\Delta_{n}\left(t_{j}\right)= \begin{cases}2 & (j=n) \\ 0 & (j \neq n)\end{cases}
$$

Then we compute

$$
\begin{aligned}
\sum_{k=0}^{n} \Delta_{n}\left(c_{k}^{(n)}\right) & =\Delta_{n}\left(\prod_{i=1}^{n}\left(1+t_{i}\right)\right) \\
& =\Delta_{n}\left(\prod_{i=1}^{n-1}\left(1+t_{i}\right)\right)\left(1+t_{n}\right)+s_{n}\left(\prod_{i=1}^{n-1}\left(1+t_{i}\right)\right) \Delta_{n}\left(1+t_{n}\right) \\
& =2 \prod_{i=1}^{n-1}\left(1+t_{i}\right)=2 \sum_{k=0}^{n-1} c_{k}^{(n-1)}
\end{aligned}
$$

by (3.2) and (4.6). From this, (2) follows. (4) follows from a similar computation.

By this lemma, we deduce that

$$
\Delta_{w}\left(c_{k}\right)= \begin{cases}2 & \text { if } w=s_{n-k+1} \cdots s_{n-1} s_{n} \\ 0 & \text { otherwise }\end{cases}
$$

Therefore, for $1 \leq k \leq n$, in $H^{*}\left(S O(2 n+1) / T^{n} ; \mathbb{Z}\right)$ we have

$$
c_{k}=2 Z_{n-k+1, \ldots, n-1, n} .
$$

Since $\gamma_{k}$ is defined by $c_{k}=2 \gamma_{k}$ and $H^{*}\left(S O(2 n+1) / T^{n} ; \mathbb{Z}\right)$ is torsion free, we see that

$$
\gamma_{k}=Z_{n-k+1, \ldots, n-1, n} .
$$

Consequently, we obtain the following result.

Proposition 4.3. In Theorem 4.1, the relation between the ring generators and the Schubert classes is given by

$$
\begin{aligned}
t_{1} & =Z_{1}, \\
t_{i} & =-Z_{i-1}+Z_{i} \quad(2 \leq i \leq n-1), \\
t_{n} & =-Z_{n-1}+2 Z_{n}, \\
\gamma_{k} & =Z_{n-k+1, \ldots, n-1, n} \quad(1 \leq k \leq n) .
\end{aligned}
$$


In particular, we can take $Z_{1}, \ldots, Z_{n}, Z_{n-1, n}, \ldots, Z_{12 \cdots n-1, n}$ as the ring generators of $H^{*}\left(S O(2 n+1) / T^{n} ; \mathbb{Z}\right)$.

REMARK 4.4. The standard projection $p$ from $S O(2 n+1) / T^{n}$ to $S O(2 n+1) / U(n)$ induces an injection

$$
p^{*}: H^{*}(S O(2 n+1) / U(n) ; \mathbb{Z}) \hookrightarrow H^{*}\left(S O(2 n+1) / T^{n} ; \mathbb{Z}\right) .
$$

The cohomology ring $H^{*}(S O(2 n+1) / U(n) ; \mathbb{Z})$ has a $\mathbb{Z}$-basis of Schubert classes $\left\{\sigma_{\lambda}\right\}$ indexed by strict partitions $\lambda$ contained in the "staircase" $\varrho_{n}=$ $(n, n-1, \ldots, 1)$. Observe that the generators $Z_{n-k+1, \ldots, n-1, n}(1 \leq k \leq n)$ in Proposition 4.3 are the $p^{*}$-images of the "special Schubert classes" $\sigma_{(k)}(1 \leq$ $k \leq n)$ that were used by P. Pragacz to describe the cohomology ring of $S O(2 n+1) / U(n)$ (see [17, Theorem 6.17]).

The case of the even special orthogonal group $S O(2 n)$ is almost identical to that of $S O(2 n+1)$. So we only exhibit the data and results. Let

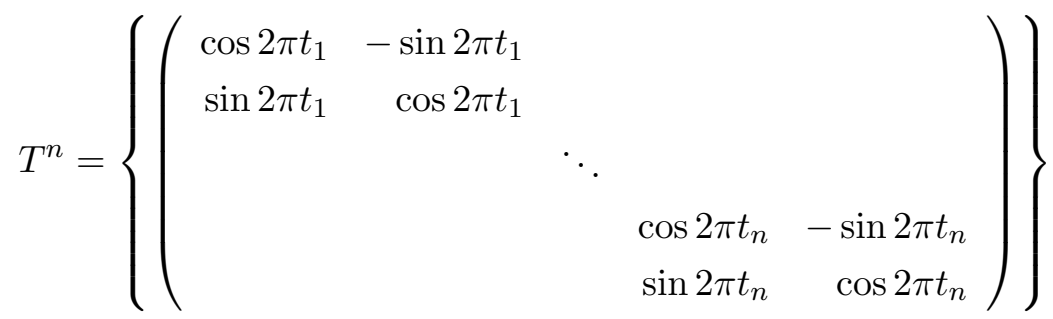

be the standard maximal torus of $S O(2 n)$. Then we have an isomorphism

$$
H^{*}\left(B T^{n} ; \mathbb{Z}\right)=\mathbb{Z}\left[t_{1}, \ldots, t_{n}\right] .
$$

The system of simple roots is

$$
\Pi=\left\{\alpha_{1}=t_{1}-t_{2}, \alpha_{2}=t_{2}-t_{3}, \ldots, \alpha_{n-1}=t_{n-1}-t_{n}, \alpha_{n}=t_{n-1}+t_{n}\right\} .
$$

The corresponding fundamental weights $\left\{\omega_{i}\right\}_{1 \leq i \leq n}$ are

$$
\begin{aligned}
\omega_{i} & =t_{1}+\cdots+t_{i} \quad(1 \leq i \leq n-2), \\
\omega_{n-1} & =\frac{1}{2}\left(t_{1}+\cdots+t_{n-1}-t_{n}\right), \\
\omega_{n} & =\frac{1}{2}\left(t_{1}+\cdots+t_{n-1}+t_{n}\right) .
\end{aligned}
$$

Let $s_{i}(1 \leq i \leq n)$ be the reflection corresponding to $\alpha_{i}(1 \leq i \leq n)$. Then $W(S O(2 n))$ is finite and is generated by $s_{i}(1 \leq i \leq n)$ which act on $\left\{t_{i}\right\}_{1 \leq i \leq n}$ as permutations and an even number of sign changes:

$$
W(S O(2 n))=\left\langle s_{1}, \ldots, s_{n}\right\rangle \cong \mathcal{S}_{n} \ltimes(\mathbb{Z} / 2 \mathbb{Z})^{n-1} .
$$

The Borel presentation of $H^{*}\left(S O(2 n) / T^{n} ; \mathbb{Z}\right)$ is given by 
Theorem 4.5 (Toda-Watanabe [19, Corollary 2.2]). The integral cohomology ring of $S O(2 n) / T^{n}$ is

$$
\begin{aligned}
H^{*}\left(S O(2 n) / T^{n} ; \mathbb{Z}\right)= & \mathbb{Z}\left[t_{1}, \ldots, t_{n}, \gamma_{1}, \ldots, \gamma_{n-1}\right] / \\
& \left(\begin{array}{c}
c_{i}-2 \gamma_{i}(1 \leq i \leq n-1), c_{n} \\
\gamma_{2 k}+\sum_{i=1}^{2 k-1}(-1)^{i} \gamma_{i} \gamma_{2 k-i}(1 \leq k \leq n-1)
\end{array}\right),
\end{aligned}
$$

where we denote by the same symbols $t_{i} \in H^{2}\left(S O(2 n) / T^{n} ; \mathbb{Z}\right)$ the images of $t_{i} \in H^{2}\left(B T^{n} ; \mathbb{Z}\right)$ under the homomorphism $c, c_{i}=e_{i}\left(t_{1}, \ldots, t_{n}\right)(1 \leq i \leq n)$, and $\gamma_{i}=0$ for $i \geq n$.

By (4.7), we have

$$
\begin{aligned}
t_{1} & =\omega_{1}, \\
t_{i} & =-\omega_{i-1}+\omega_{i} \quad(2 \leq i \leq n-2), \\
t_{n-1} & =-\omega_{n-2}+\omega_{n-1}+\omega_{n}, \\
t_{n} & =-\omega_{n-1}+\omega_{n} .
\end{aligned}
$$

Since $c\left(\omega_{i}\right)=Z_{i}$, it follows from (4.9) that

$$
\begin{aligned}
t_{1} & =Z_{1}, \\
t_{i} & =-Z_{i-1}+Z_{i} \quad(2 \leq i \leq n-2), \\
t_{n-1} & =-Z_{n-2}+Z_{n-1}+Z_{n}, \\
t_{n} & =-Z_{n-1}+Z_{n} .
\end{aligned}
$$

By (4.9), we have, for $1 \leq i \leq n-1$,

$$
\Delta_{i}\left(t_{j}\right)= \begin{cases}1 & (j=i), \\ -1 & (j=i+1), \\ 0 & (j \neq i, i+1)\end{cases}
$$

and

$$
\Delta_{n}\left(t_{j}\right)= \begin{cases}1 & (j=n-1), \\ 1 & (j=n), \\ 0 & (j \neq n-1, n) .\end{cases}
$$

Then we obtain the following quite similarly to Lemma 4.2.

Lemma 4.6. For fixed $k, 1 \leq k \leq n-1$, we have

(1) $\Delta_{i}\left(c_{k}^{(n)}\right)=0(1 \leq i \leq n-1)$,

(2) $\Delta_{n}\left(c_{k}^{(n)}\right)=2 c_{k-1}^{(n-2)}$,

(3) $\Delta_{i}\left(c_{k-j+1}^{(n-j)}\right)=0(1 \leq i \leq n-j-1,2 \leq j \leq n-1)$,

(4) $\Delta_{n-j}\left(c_{k-j+1}^{(n-j)}\right)=c_{k-j}^{(n-j-1)}(2 \leq j \leq n-1)$. 
By this lemma, we deduce that

$$
\begin{aligned}
& \Delta_{w}\left(c_{1}\right)= \begin{cases}2 & \text { if } w=s_{n} \\
0 & \text { otherwise }\end{cases} \\
& \Delta_{w}\left(c_{k}\right)= \begin{cases}2 & \text { if } w=s_{n-k} \cdots s_{n-2} s_{n} \\
0 & \text { otherwise }\end{cases}
\end{aligned}
$$

for $2 \leq k \leq n-1$. Therefore

$$
c_{1}=2 Z_{n}, \quad c_{k}=2 Z_{n-k, \ldots, n-2, n} \quad(2 \leq k \leq n-1)
$$

in $H^{*}\left(S O(2 n) / T^{n} ; \mathbb{Z}\right)$. Since $\gamma_{k}$ is defined by $c_{k}=2 \gamma_{k}$ and $H^{*}\left(S O(2 n) / T^{n} ; \mathbb{Z}\right)$ is torsion free, we see that

$$
\gamma_{1}=Z_{n}, \quad \gamma_{k}=Z_{n-k, \ldots, n-2, n} \quad(2 \leq k \leq n-1) .
$$

Consequently, we obtain the following result.

Proposition 4.7. In Theorem 4.5, the relation between the ring generators and the Schubert classes is given by

$$
\begin{aligned}
t_{1} & =Z_{1}, \\
t_{i} & =-Z_{i-1}+Z_{i} \quad(2 \leq i \leq n-2), \\
t_{n-1} & =-Z_{n-2}+Z_{n-1}+Z_{n}, \\
t_{n} & =-Z_{n-1}+Z_{n}, \\
\gamma_{1} & =Z_{n}, \\
\gamma_{k} & =Z_{n-k, \ldots, n-2, n} \quad(2 \leq k \leq n-1) .
\end{aligned}
$$

In particular, we can take $Z_{1}, \ldots, Z_{n}, Z_{n-2, n}, \ldots, Z_{12 \cdots n-2, n}$ as the ring generators of $H^{*}\left(S O(2 n) / T^{n} ; \mathbb{Z}\right)$.

REMARK 4.8. The standard projection $p: S O(2 n) / T^{n} \rightarrow S O(2 n) / U(n)$ induces an injection

$$
p^{*}: H^{*}(S O(2 n) / U(n) ; \mathbb{Z}) \hookrightarrow H^{*}\left(S O(2 n) / T^{n} ; \mathbb{Z}\right) .
$$

The cohomology ring $H^{*}(S O(2 n) / U(n) ; \mathbb{Z})$ has a $\mathbb{Z}$-basis of Schubert classes $\left\{\sigma_{\lambda}\right\}$ indexed by strict partitions $\lambda$ contained in $\varrho_{n-1}=(n-1, \ldots, 1)$. Observe that the generators $Z_{n}, Z_{n-k, \ldots, n-2, n}(2 \leq k \leq n-1)$ in Proposition 4.7 are the $p^{*}$-images of the special Schubert classes $\sigma_{(k)}(1 \leq k \leq$ $n-1)$ that were also used by P. Pragacz to describe the cohomology ring of $S O(2 n) / U(n)$ (see $\left[17\right.$, Theorem $\left.\left.6.17^{\prime}\right]\right)$.

5. The case of $G_{2}$. In this section, we concentrate on the exceptional Lie group $G_{2}$. Let $T$ be a maximal torus of $G_{2}$. Following [4], we take the system of simple roots $\Pi=\left\{\alpha_{1}, \alpha_{2}\right\}$ and the corresponding fundamental weights $\left\{\omega_{1}, \omega_{2}\right\}$. Then we can identify

$$
H^{*}(B T ; \mathbb{Z})=\mathbb{Z}\left[\omega_{1}, \omega_{2}\right] .
$$


Let $s_{i}(i=1,2)$ be the reflection corresponding to the simple root $\alpha_{i}$ $(i=1,2)$. Then the Weyl group $W\left(G_{2}\right)$ of $G_{2}$ is finite and is generated by $s_{i}(i=1,2)$ :

$$
W\left(G_{2}\right)=\left\langle s_{1}, s_{2}\right\rangle, \quad s_{1}^{2}=s_{2}^{2}=1,\left(s_{1} s_{2}\right)^{6}=1 .
$$

Now we review the Borel presentation of $H^{*}\left(G_{2} / T ; \mathbb{Z}\right)$. We put

$$
\begin{aligned}
& t_{1}=-\omega_{1}, \quad t_{2}=-\omega_{1}+\omega_{2}, \quad t_{3}=2 \omega_{1}-\omega_{2}, \\
& c_{i}=e_{i}\left(t_{1}, t_{2}, t_{3}\right) .
\end{aligned}
$$

Then we can write

$$
H^{*}(B T ; \mathbb{Z})=\mathbb{Z}\left[t_{1}, t_{2}, t_{3}\right] /\left(c_{1}\right) .
$$

The action of $W\left(G_{2}\right)$ on $\left\{t_{1}, t_{2}, t_{3}\right\}$ is given by Table 1 .

\section{Table 1}

\begin{tabular}{c|cc} 
& $s_{1}$ & $s_{2}$ \\
\hline$t_{1}$ & $-t_{2}$ & $t_{1}$ \\
$t_{2}$ & $-t_{1}$ & $t_{3}$ \\
$t_{3}$ & $-t_{3}$ & $t_{2}$
\end{tabular}

REMARK 5.1. The elements $\left\{t_{i}\right\}_{i=1,2,3}$ are derived from the natural inclusion $T \subset S U(3) \subset G_{2}$.

The integral cohomology ring of $G_{2} / T$ was first determined by BottSamelson [3], but we prefer to use the presentation due to Toda-Watanabe.

Theorem 5.2 (Bott-Samelson [3], Toda-Watanabe [19]). The integral cohomology ring of $G_{2} / T$ is

$$
H^{*}\left(G_{2} / T ; \mathbb{Z}\right)=\mathbb{Z}\left[t_{1}, t_{2}, t_{3}, \gamma_{3}\right] /\left(\varrho_{1}, \varrho_{2}, \varrho_{3}, \varrho_{6}\right),
$$

where $\varrho_{1}=c_{1}, \varrho_{2}=c_{2}, \varrho_{3}=c_{3}-2 \gamma_{3}, \varrho_{6}=\gamma_{3}^{2}$, and we denote by the same symbols $t_{i} \in H^{2}\left(G_{2} / T ; \mathbb{Z}\right)$ the images of $t_{i} \in H^{2}(B T ; \mathbb{Z})$ under the homomorphism $c$.

By (5.1), the elements of the Weyl group $W\left(G_{2}\right)$ are given by the following table.

\begin{tabular}{lll}
\hline$l(w)$ & \multicolumn{2}{c}{ Elements of $W\left(G_{2}\right)$} \\
\hline 0 & 1 & $s_{2}$ \\
1 & $s_{1}$ & $s_{2} s_{1}$ \\
2 & $s_{1} s_{2}$ & $s_{2} s_{1} s_{2}$ \\
3 & $s_{1} s_{2} s_{1}$ & $s_{2} s_{1} s_{2} s_{1}$ \\
4 & $s_{1} s_{2} s_{1} s_{2}$ & $s_{2} s_{1} s_{2} s_{1} s_{2}$ \\
5 & $s_{1} s_{2} s_{1} s_{2} s_{1}$ & \\
6 & $s_{1} s_{2} s_{1} s_{2} s_{1} s_{2}$ & \\
& &
\end{tabular}


Therefore the corresponding Schubert basis for $H^{*}\left(G_{2} / T ; \mathbb{Z}\right)$ is as follows:

\begin{tabular}{cccccccc}
\hline $\operatorname{deg}$ & 0 & 2 & 4 & 6 & 8 & 10 & 12 \\
\hline & 1 & $Z_{1}$ & $Z_{12}$ & $Z_{121}$ & $Z_{1212}$ & $Z_{12121}$ & \\
& $Z_{2}$ & $Z_{21}$ & $Z_{212}$ & $Z_{2121}$ & $Z_{21212}$ & $Z_{121212}$ \\
\hline
\end{tabular}

Here we denote $Z_{s_{i}}$ simply by $Z_{i}$ and so on. We wish to express the algebra generators $\left\{t_{1}, t_{2}, t_{3}, \gamma_{3}\right\}$ in terms of Schubert classes. Since $c\left(\omega_{i}\right)=Z_{i}(i=$ $1,2)$, it follows from $(5.2)$ that

$$
t_{1}=-Z_{1}, \quad t_{2}=-Z_{1}+Z_{2}, \quad t_{3}=2 Z_{1}-Z_{2} .
$$

Next we can put

$$
\gamma_{3}=a_{121} Z_{121}+a_{212} Z_{212}
$$

for some integers $a_{121}, a_{212}$ and we need to determine the coefficients $a_{121}, a_{212}$. The characteristic homomorphism $c$ is not surjective over $\mathbb{Z}$ and $\gamma_{3}$ is not in its image, but $2 \gamma_{3}=c_{3}$ is. Thus putting

$$
c_{3}=b_{121} Z_{121}+b_{212} Z_{212}
$$

for some integers $b_{121}, b_{212}$, we can compute the coefficients $b_{121}, b_{212}$ using the divided difference operators. By (5.2), we have

$$
c_{3}=t_{1} t_{2} t_{3}=2 \omega_{1}^{3}-3 \omega_{1}^{2} \omega_{2}+\omega_{1} \omega_{2}^{2} .
$$

Therefore we derive

$$
\begin{aligned}
& b_{121}=\Delta_{1} \Delta_{2} \Delta_{1}\left(c_{3}\right)=\Delta_{1} \Delta_{2} \Delta_{1}\left(2 \omega_{1}^{3}-3 \omega_{1}^{2} \omega_{2}+\omega_{1} \omega_{2}^{2}\right)=-2, \\
& b_{212}=\Delta_{2} \Delta_{1} \Delta_{2}\left(c_{3}\right)=\Delta_{2} \Delta_{1} \Delta_{2}\left(2 \omega_{1}^{3}-3 \omega_{1}^{2} \omega_{2}+\omega_{1} \omega_{2}^{2}\right)=0
\end{aligned}
$$

from (3.1), (3.2) and Table 1. Thus we have $c_{3}=-2 Z_{121}$ in $H^{6}\left(G_{2} / T ; \mathbb{Z}\right)$. Since $\gamma_{3}$ is defined by $c_{3}=2 \gamma_{3}$ and $H^{*}\left(G_{2} / T ; \mathbb{Z}\right)$ is torsion free, we see that $\gamma_{3}=-Z_{121}$. Consequently, we obtain the following result.

Proposition 5.3. In Theorem 5.2, the relation between the ring generators $\left\{t_{1}, t_{2}, t_{3}, \gamma_{3}\right\}$ and the Schubert classes is given by

$$
\begin{aligned}
t_{1} & =-Z_{1}, \quad t_{2}=-Z_{1}+Z_{2}, \quad t_{3}=2 Z_{1}-Z_{2}, \\
\gamma_{3} & =-Z_{121} .
\end{aligned}
$$

In particular, we can take $Z_{1}, Z_{2}, Z_{121}$ as the ring generators of $H^{*}\left(G_{2} / T ; \mathbb{Z}\right)$.

6. The case of $F_{4}$. In this section, we deal with the exceptional Lie group $F_{4}$. Let $T$ be a maximal torus of $F_{4}$. Following [4], we take the system of simple roots $\Pi=\left\{\alpha_{i}\right\}_{1 \leq i \leq 4}$ and the corresponding fundamental weights $\left\{\omega_{i}\right\}_{1 \leq i \leq 4}$. Then we can identify

$$
H^{*}(B T ; \mathbb{Z})=\mathbb{Z}\left[\omega_{1}, \omega_{2}, \omega_{3}, \omega_{4}\right] .
$$


Let $s_{i}(1 \leq i \leq 4)$ be the reflection corresponding to $\alpha_{i}(1 \leq i \leq 4)$. Then $W\left(F_{4}\right)$ is finite and

$$
\begin{aligned}
& W\left(F_{4}\right)=\left\langle s_{1}, s_{2}, s_{3}, s_{4}\right\rangle, \\
& s_{1}^{2}=s_{2}^{2}=s_{3}^{2}=s_{4}^{2}=1, \\
& \left(s_{1} s_{2}\right)^{3}=\left(s_{3} s_{4}\right)^{3}=\left(s_{2} s_{3}\right)^{4}=1, \\
& s_{1} s_{3}=s_{3} s_{1}, \quad s_{1} s_{4}=s_{4} s_{1}, \quad s_{2} s_{4}=s_{4} s_{2} .
\end{aligned}
$$

Now we review the Borel presentation of $H^{*}\left(F_{4} / T ; \mathbb{Z}\right)$. We put

$$
\begin{aligned}
t_{1} & =-\omega_{4}, \\
t_{2} & =\omega_{1}-\omega_{4}, \\
t_{3} & =-\omega_{1}+\omega_{2}-\omega_{4}, \\
t_{4} & =-\omega_{2}+2 \omega_{3}-\omega_{4}, \\
c_{i} & =e_{i}\left(t_{1}, \ldots, t_{4}\right), \\
t & =\frac{1}{2} c_{1}=\omega_{3}-2 \omega_{4} .
\end{aligned}
$$

Then we can write

$$
H^{*}(B T ; \mathbb{Z})=\mathbb{Z}\left[t_{1}, t_{2}, t_{3}, t_{4}, t\right] /\left(c_{1}-2 t\right) .
$$

The action of $W\left(F_{4}\right)$ on $\left\{t_{i}\right\}_{1 \leq i \leq 4}$ is given by Table 2 , where blanks indicate the trivial action.

\section{Table 2}

\begin{tabular}{c|cccc} 
& $s_{1}$ & $s_{2}$ & $s_{3}$ & $s_{4}$ \\
\hline$t_{1}$ & & & & $t_{1}-t$ \\
$t_{2}$ & $t_{3}$ & & & $t_{2}-t$ \\
$t_{3}$ & $t_{2}$ & $t_{4}$ & & $t_{3}-t$ \\
$t_{4}$ & & $t_{3}$ & $-t_{4}$ & $t_{4}-t$ \\
$t$ & & & $t-t_{4}$ & $-t$
\end{tabular}

REMARK 6.1. The elements $\left\{t_{i}\right\}_{1 \leq i \leq 4}$ and $t$ are derived from the natural inclusion $T \subset \operatorname{Spin}(9) \subset F_{4}$.

The integral cohomology ring of $F_{4} / T$ was determined by Toda-Watanabe [19].

Theorem 6.2 (Toda-Watanabe [19, Theorem A]). The integral cohomology ring of $F_{4} / T$ is

$$
H^{*}\left(F_{4} / T ; \mathbb{Z}\right)=\mathbb{Z}\left[t_{1}, t_{2}, t_{3}, t_{4}, t, \gamma_{3}, \gamma_{4}\right] /\left(\varrho_{1}, \varrho_{2}, \varrho_{3}, \varrho_{4}, \varrho_{6}, \varrho_{8}, \varrho_{12}\right),
$$

where

$$
\varrho_{1}=c_{1}-2 t, \quad \varrho_{2}=c_{2}-2 t^{2}, \quad \varrho_{3}=c_{3}-2 \gamma_{3},
$$




$$
\begin{aligned}
\varrho_{4} & =c_{4}-4 t \gamma_{3}+8 t^{4}-3 \gamma_{4}, \\
\varrho_{6} & =\gamma_{3}^{2}-3 t^{2} \gamma_{4}-4 t^{3} \gamma_{3}+8 t^{6} \\
\varrho_{8} & =3 \gamma_{4}^{2}+6 t \gamma_{3} \gamma_{4}-3 t^{4} \gamma_{4}-13 t^{8}, \\
\varrho_{12} & =\gamma_{4}^{3}-6 t^{4} \gamma_{4}^{2}+12 t^{8} \gamma_{4}-8 t^{12},
\end{aligned}
$$

and we denote by the same symbols $t_{i} \in H^{2}\left(F_{4} / T ; \mathbb{Z}\right)$ the images of $t_{i} \in$ $H^{2}(B T ; \mathbb{Z})$ under $c$.

REMARK 6.3. In [19], Toda and Watanabe described $H^{*}\left(F_{4} / T ; \mathbb{Z}\right)$ using the inclusion $H^{*}\left(F_{4} / \operatorname{Spin}(9) ; \mathbb{Z}\right) \hookrightarrow H^{*}\left(F_{4} / T ; \mathbb{Z}\right)$ and the known structure of $H^{*}\left(F_{4} / \operatorname{Spin}(9) ; \mathbb{Z}\right)$. Theorem 6.2 is a rewritten form of their result in terms of the $W\left(F_{4}\right)$-invariants.

By (6.1), the elements of the Weyl group $W\left(F_{4}\right)$ of length $\leq 4$ are given by the following table.

\begin{tabular}{llllll}
\hline$l(w)$ & \multicolumn{5}{c}{ Elements of $W\left(F_{4}\right)$} \\
\hline 0 & 1 & & & & \\
1 & $s_{1}$ & $s_{2}$ & $s_{3}$ & $s_{4}$ & \\
2 & $s_{1} s_{2}$ & $s_{1} s_{3}$ & $s_{1} s_{4}$ & $s_{2} s_{1}$ & $s_{2} s_{3}$ \\
& $s_{2} s_{4}$ & $s_{3} s_{2}$ & $s_{3} s_{4}$ & $s_{4} s_{3}$ & \\
3 & $s_{1} s_{2} s_{1}$ & $s_{1} s_{2} s_{3}$ & $s_{1} s_{2} s_{4}$ & $s_{1} s_{3} s_{2}$ & $s_{1} s_{3} s_{4}$ \\
& $s_{1} s_{4} s_{3}$ & $s_{2} s_{1} s_{3}$ & $s_{2} s_{1} s_{4}$ & $s_{2} s_{3} s_{2}$ & $s_{2} s_{3} s_{4}$ \\
& $s_{2} s_{4} s_{3}$ & $s_{3} s_{2} s_{1}$ & $s_{3} s_{2} s_{3}$ & $s_{3} s_{2} s_{4}$ & $s_{3} s_{4} s_{3}$ \\
& $s_{4} s_{3} s_{2}$ & & & & \\
4 & $s_{1} s_{2} s_{1} s_{3}$ & $s_{1} s_{2} s_{1} s_{4}$ & $s_{1} s_{2} s_{3} s_{2}$ & $s_{1} s_{2} s_{3} s_{4}$ & $s_{1} s_{2} s_{4} s_{3}$ \\
& $s_{1} s_{3} s_{2} s_{1}$ & $s_{1} s_{3} s_{2} s_{3}$ & $s_{1} s_{3} s_{2} s_{4}$ & $s_{1} s_{3} s_{4} s_{3}$ & $s_{1} s_{4} s_{3} s_{2}$ \\
& $s_{2} s_{1} s_{3} s_{2}$ & $s_{2} s_{1} s_{3} s_{4}$ & $s_{2} s_{1} s_{4} s_{3}$ & $s_{2} s_{3} s_{2} s_{1}$ & $s_{2} s_{3} s_{2} s_{3}$ \\
& $s_{2} s_{3} s_{2} s_{4}$ & $s_{2} s_{3} s_{4} s_{3}$ & $s_{2} s_{4} s_{3} s_{2}$ & $s_{3} s_{2} s_{1} s_{3}$ & $s_{3} s_{2} s_{1} s_{4}$ \\
& $s_{3} s_{2} s_{3} s_{4}$ & $s_{3} s_{2} s_{4} s_{3}$ & $s_{3} s_{4} s_{3} s_{2}$ & $s_{4} s_{3} s_{2} s_{1}$ & $s_{4} s_{3} s_{2} s_{3}$ \\
\hline
\end{tabular}

We have the corresponding Schubert basis $\left\{Z_{w}\right\}_{w \in W\left(F_{4}\right)}$. As before, we denote $Z_{s_{i}}$ simply by $Z_{i}$ and so on. We wish to express the algebra generators $\left\{t_{1}, t_{2}, t_{3}, t_{4}, t, \gamma_{3}, \gamma_{4}\right\}$ in terms of Schubert classes. Since $c\left(\omega_{i}\right)=Z_{i}$, it follows from (6.2) that

$$
\begin{aligned}
t_{1} & =-Z_{4}, \\
t_{2} & =Z_{1}-Z_{4}, \\
t_{3} & =-Z_{1}+Z_{2}-Z_{4}, \\
t_{4} & =-Z_{2}+2 Z_{3}-Z_{4}, \\
t & =Z_{3}-2 Z_{4} .
\end{aligned}
$$


Next we can put

$$
\gamma_{3}=\sum_{l(w)=3} a_{w} Z_{w}, \quad \gamma_{4}=\sum_{l(w)=4} a_{w} Z_{w}
$$

for some integers $a_{w}$. We wish to determine the coefficients $a_{w}$. By Theorem 6.2 , we have

$$
\begin{aligned}
& 2 \gamma_{3}=c_{3}, \\
& 3 \gamma_{4}=c_{4}-4 t \gamma_{3}+8 t^{4}=c_{4}-2 t c_{3}+8 t^{4} .
\end{aligned}
$$

Therefore $2 \gamma_{3}$ and $3 \gamma_{4}$ are contained in the image of $c$. So as in the case of $G_{2}$, we apply the divided difference operators to the right hand side of (6.4). The result can be seen in the following table.

\begin{tabular}{ccccccccc}
\hline & $\Delta_{121}$ & $\Delta_{123}$ & $\Delta_{124}$ & $\Delta_{132}$ & $\Delta_{134}$ & $\Delta_{143}$ & $\Delta_{213}$ & $\Delta_{214}$ \\
$c_{3}$ & 0 & 2 & 0 & 0 & 0 & 0 & 0 & 0 \\
\hline & $\Delta_{232}$ & $\Delta_{234}$ & $\Delta_{243}$ & $\Delta_{321}$ & $\Delta_{323}$ & $\Delta_{324}$ & $\Delta_{343}$ & $\Delta_{432}$ \\
$c_{3}$ & 0 & -2 & -4 & 0 & 0 & 0 & 6 & 0 \\
\hline
\end{tabular}

Here we denote $\Delta_{1} \Delta_{2} \Delta_{1}$ simply by $\Delta_{121}$ and so on.

Thus we have

$$
\begin{aligned}
c_{3} & =2 Z_{123}-2 Z_{234}-4 Z_{243}+6 Z_{343} \\
& =2\left(Z_{123}-Z_{234}-2 Z_{243}+3 Z_{343}\right)
\end{aligned}
$$

in $H^{6}\left(F_{4} / T ; \mathbb{Z}\right)$. Since $\gamma_{3}$ is defined by $c_{3}=2 \gamma_{3}$ and $H^{*}\left(F_{4} / T ; \mathbb{Z}\right)$ is torsion free, we see that

$$
\gamma_{3}=Z_{123}-Z_{234}-2 Z_{243}+3 Z_{343} .
$$

Similarly we obtain

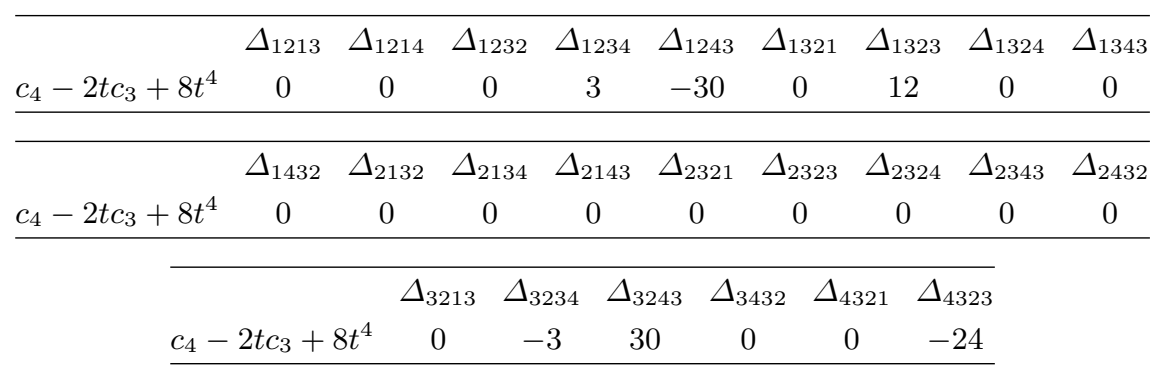

Thus we have

$$
\begin{aligned}
c_{4}-2 t c_{3}+8 t^{4} & =3 Z_{1234}-30 Z_{1243}+12 Z_{1323}-3 Z_{3234}+30 Z_{3243}-24 Z_{4323} \\
& =3\left(Z_{1234}-10 Z_{1243}+4 Z_{1323}-Z_{3234}+10 Z_{3243}-8 Z_{4323}\right)
\end{aligned}
$$


in $H^{8}\left(F_{4} / T ; \mathbb{Z}\right)$. Since $\gamma_{4}$ is defined by $c_{4}-2 t c_{3}+8 t^{4}=3 \gamma_{4}$ and $H^{*}\left(F_{4} / T ; \mathbb{Z}\right)$ is torsion free, we see that

$$
\gamma_{4}=Z_{1234}-10 Z_{1243}+4 Z_{1323}-Z_{3234}+10 Z_{3243}-8 Z_{4323} \text {. }
$$

Unfortunately, from (6.5) and (6.6), we cannot decide which Schubert classes are indecomposable. This leads us to the use of the Giambelli formula. We need the following data.

(1) A set of positive roots of $F_{4}$ is given by

$$
\Delta^{+}=\left\{\begin{array}{l}
t_{i} \pm t_{j}(1 \leq i<j \leq 4), \\
t_{i}(1 \leq i \leq 4), \frac{1}{2}\left(t_{1} \pm t_{2} \pm t_{3} \pm t_{4}\right)
\end{array}\right\} .
$$

(2) The longest element $w_{0}$ of the Weyl group $W\left(F_{4}\right)$ is given by

$$
w_{0}=s_{1} s_{2} s_{1} s_{3} s_{2} s_{1} s_{3} s_{2} s_{3} s_{4} s_{3} s_{2} s_{1} s_{3} s_{2} s_{3} s_{4} s_{3} s_{2} s_{1} s_{3} s_{2} s_{3} s_{4} .
$$

Then, using the Giambelli formula (Theorem 3.3), we obtain the following result.

Lemma 6.4. In (6.5) and (6.6), each Schubert class is expressed in terms of $t_{i}(1 \leq i \leq 4), t, \gamma_{3}, \gamma_{4}$ as follows:

$$
\begin{aligned}
Z_{123} & =\gamma_{3}-2 t_{1}^{3}+3 t_{1}^{2} t-2 t_{1} t^{2}, \\
Z_{234} & =-t_{1}^{3}, \\
Z_{243} & =-2 t_{1}^{3}+3 t_{1}^{2} t-t_{1} t^{2} \\
Z_{343} & =-t_{1}^{3}+t_{1}^{2} t \\
Z_{1234} & =-\gamma_{4}+\left(t_{1}-2 t\right) \gamma_{3}+t_{1}^{3} t-t_{1}^{2} t^{2}+3 t^{4}, \\
Z_{1243} & =\gamma_{4}+\left(-2 t_{1}+2 t\right) \gamma_{3}+2 t_{1}^{4}-4 t_{1}^{3} t+3 t_{1}^{2} t^{2}-3 t^{4}, \\
Z_{1323} & =-\gamma_{4}-t \gamma_{3}+2 t_{1}^{4}-4 t_{1}^{3} t+4 t_{1}^{2} t^{2}-2 t_{1} t^{3}+3 t^{4}, \\
Z_{3234} & =\gamma_{4}+\left(-t_{1}+2 t\right) \gamma_{3}+t_{1}^{4}-t_{1}^{3} t+t_{1}^{2} t^{2}-3 t^{4}, \\
Z_{3243} & =t_{1}^{4}-2 t_{1}^{3} t+t_{1}^{2} t^{2}, \\
Z_{4323} & =-\gamma_{4}+\left(2 t_{1}-2 t\right) \gamma_{3}-t_{1} t^{3}+3 t^{4} .
\end{aligned}
$$

In particular, $Z_{123}$ and $Z_{1234}$ are indecomposable in the ring $H^{*}\left(F_{4} / T ; \mathbb{Z}\right)$.

Consequently, we obtain the following result.

Proposition 6.5. In Theorem 6.2, the relation between the ring generators $\left\{t_{1}, t_{2}, t_{3}, t_{4}, t, \gamma_{3}, \gamma_{4}\right\}$ and the Schubert classes is given by

$$
\begin{aligned}
& t_{1}=-Z_{4}, \\
& t_{2}=Z_{1}-Z_{4}, \\
& t_{3}=-Z_{1}+Z_{2}-Z_{4}, \\
& t_{4}=-Z_{2}+2 Z_{3}-Z_{4},
\end{aligned}
$$




$$
\begin{aligned}
t & =Z_{3}-2 Z_{4}, \\
\gamma_{3} & =Z_{123}-Z_{234}-2 Z_{243}+3 Z_{343}, \\
\gamma_{4} & =Z_{1234}-10 Z_{1243}+4 Z_{1323}-Z_{3234}+10 Z_{3243}-8 Z_{4323} .
\end{aligned}
$$

Furthermore,

$$
\begin{aligned}
Z_{123} & =\gamma_{3}-2 t_{1}^{3}+3 t_{1}^{2} t-2 t_{1} t^{2}, \\
Z_{1234} & =-\gamma_{4}+\left(t_{1}-2 t\right) \gamma_{3}+t_{1}^{3} t-t_{1}^{2} t^{2}+3 t^{4},
\end{aligned}
$$

and we can take $Z_{1}, Z_{2}, Z_{3}, Z_{4}, Z_{123}, Z_{1234}$ as the ring generators of $H^{*}\left(F_{4} / T ; \mathbb{Z}\right)$.

7. The Chow rings of $\mathrm{SO}(n), \operatorname{Spin}(n), \mathrm{G}_{2}$ and $\mathrm{F}_{4}$. In this section, using our description of the integral cohomology rings of the flag manifolds of types $B_{n}, D_{n}, G_{2}$ and $F_{4}$ (see Sections 4-6) and a remark of Grothendieck, we compute the Chow rings of the corresponding complex algebraic groups.

As in Section 2, let $K$ be a compact simply connected simple Lie group, $T$ its maximal torus, $G=K^{\mathbb{C}}$ the complexification of $K$ and $B$ a Borel subgroup of $G$ containing $T$. Let $A^{i}(\cdot)$ denote the Chow group of codimension $i$ cycles up to rational equivalence and $A(\cdot)=\bigoplus_{i>0} A^{i}(\cdot)$. Given any character $\chi$ of $B$, that is, a homomorphism of $B$ into $\mathbb{C}^{\times}$, we have the associated line bundle $L_{\chi}$ over $G / B$, which defines an element of $A^{1}(G / B)$, denoted by $c(\chi)$. This induces a homomorphism

$$
c: \widehat{B} \rightarrow A^{1}(G / B),
$$

where $\widehat{B}$ denotes the character group of $B$. Extending this homomorphism $c$ by multiplicativity to the symmetric algebra $S(\widehat{B})$ of $\widehat{B}$, one obtains a homomorphism

$$
c: S(\widehat{B}) \rightarrow A(G / B),
$$

which is also called the characteristic homomorphism.

Then Grothendieck's remark ([9, p. 21, Remarques $\left.\left.2^{\circ}\right]\right)$ allows us to obtain $A(G)$ as the quotient of $A(G / B)$ by the ideal generated by $c(\widehat{B})$. Denote by $T_{G}: A(G / B) \rightarrow A(G)$ the canonical map onto the quotient. It is known ([9, Lemme 10]) that the Chow $\operatorname{ring} A(G / B)$ of $G / B$ is isomorphic to the integral cohomology ring $H^{*}(G / B ; \mathbb{Z})$ of $G / B$. Under this isomorphism, the Schubert variety $X_{w_{0} w}$ corresponds to the Schubert class $Z_{w}$ and the above characteristic homomorphism (7.1) coincides with the characteristic homomorphism (2.1).

Thus, in order to determine the Chow ring $A(G)$, we need only compute the quotient ring of $H^{*}(K / T ; \mathbb{Z})$ by the ideal generated by $c\left(H^{2}(B T ; \mathbb{Z})\right)$. Since $K$ is assumed to be simply connected, $H^{2}(B T ; \mathbb{Z}) \cong H^{2}(K / T ; \mathbb{Z})$. Therefore we compute the quotient ring $H^{*}(K / T ; \mathbb{Z}) /\left(H^{2}(K / T ; \mathbb{Z})\right)$. We 
will show how to do this for $F_{4}$. By Theorem 6.2 , we have

$$
H^{*}\left(F_{4} / T ; \mathbb{Z}\right) /\left(t_{1}, t_{2}, t_{3}, t_{4}, t\right)=\mathbb{Z}\left[\gamma_{3}, \gamma_{4}\right] /\left(2 \gamma_{3}, 3 \gamma_{4}, \gamma_{3}^{2}, \gamma_{4}^{3}\right) .
$$

Taking Proposition 6.5 into account, we can replace $\gamma_{3}, \gamma_{4}$ with $Z_{123}, Z_{1234}$ respectively. Thus we obtain the following

ThEOREM 7.1. If $G$ is of type $\mathrm{F}_{4}$, we have

$$
A\left(\mathrm{~F}_{4}\right)=\mathbb{Z}\left[X_{3}, X_{4}\right] /\left(2 X_{3}, 3 X_{4}, X_{3}^{2}, X_{4}^{3}\right),
$$

where $X_{3}\left(\right.$ resp. $\left.X_{4}\right)$ is the image under $T_{G}$ of the element of $A(G / B)$ defined by the Schubert variety $X_{w_{0} s_{1} s_{2} s_{3}}$ (resp. $X_{w_{0} s_{1} s_{2} s_{3} s_{4}}$ ).

In a similar way, we can compute the Chow rings of $\mathrm{SO}(n), \operatorname{Spin}(n)$ and $\mathrm{G}_{2}$. The results are summarized as follows.

THEOREM 7.2. If $G$ is of type $B_{n}(\mathrm{SO}(2 n+1)$ or $\operatorname{Spin}(2 n+1))$ and $x_{i}(1 \leq i \leq n)$ is the element of $A(G / B)$ defined by the Schubert variety $X_{w_{0} s_{n-i+1} \cdots s_{n-1} s_{n}}$. Then

$$
\begin{aligned}
A(\mathrm{SO}(2 n+1)) & =\mathbb{Z}\left[X_{1}, X_{3}, X_{5}, \ldots, X_{2[(n+1) / 2]-1}\right] /\left(2 X_{i}, X_{i}^{p_{i}}\right), \\
A(\operatorname{Spin}(2 n+1)) & =\mathbb{Z}\left[X_{3}, X_{5}, \ldots, X_{2[(n+1) / 2]-1}\right] /\left(2 X_{i}, X_{i}^{p_{i}}\right),
\end{aligned}
$$

where

$$
p_{i}=2^{\left[\log _{2}(n / i)\right]+1}, \quad X_{i}=T_{G}\left(x_{i}\right) .
$$

TheOREM 7.3. If $G$ is of type $D_{n}(\mathrm{SO}(2 n)$ or $\operatorname{Spin}(2 n))$ and $x_{1}$ (resp. $\left.x_{i}(2 \leq i \leq n-1)\right)$ is the element of $A(G / B)$ defined by the Schubert variety $X_{w_{0} s_{n}}\left(\right.$ resp. $\left.X_{w_{0} s_{n-i} \ldots s_{n-2} s_{n}}(2 \leq i \leq n-1)\right)$, then

$$
\begin{aligned}
A(\mathrm{SO}(2 n)) & =\mathbb{Z}\left[X_{1}, X_{3}, X_{5}, \ldots, X_{2[n / 2]-1}\right] /\left(2 X_{i}, X_{i}^{p_{i}}\right), \\
A(\operatorname{Spin}(2 n)) & =\mathbb{Z}\left[X_{3}, X_{5}, \ldots, X_{2[n / 2]-1}\right] /\left(2 X_{i}, X_{i}^{p_{i}}\right),
\end{aligned}
$$

where

$$
p_{i}=2^{\left[\log _{2} \frac{n-1}{i}\right]+1}, \quad X_{i}=T_{G}\left(x_{i}\right) .
$$

Theorem 7.4. If $G$ is of type $\mathrm{G}_{2}$, we have

$$
A\left(\mathrm{G}_{2}\right)=\mathbb{Z}\left[X_{3}\right] /\left(2 X_{3}, X_{3}^{2}\right),
$$

where $X_{3}$ is the image under $T_{G}$ of the element of $A(G / B)$ defined by the Schubert variety $X_{w_{0} s_{1} s_{2} s_{1}}$.

We observe that our results obtained in this section agree with those of Marlin [13].

\section{References}

[1] I. N. Bernstein, I. M. Gelfand and S. I. Gelfand, Schubert cells, and the cohomology of the spaces $G / P$, in: London Math. Soc. Lecture Note Ser. 69, Cambridge Univ. Press, 1982, 115-140. 
[2] A. Borel, Sur la cohomologie des espaces fibrés principaux et des espaces homogènes de groupes de Lie compacts, Ann. of Math. 57 (1953), 115-207.

[3] R. Bott and H. Samelson, The integral cohomology ring of $G / T$, Proc. Nat. Acad. Sci. USA 41 (1955), 490-493.

[4] N. Bourbaki, Groupes et Algèbres de Lie. Chapitre 4, 5 et 6, Masson, Paris, 1981.

[5] C. Chevalley, Sur les décompositions cellulaires des espaces $G / B$, in: Algebraic Groups and their Generalizations: Classical Methods, W. Haboush (ed.), Proc. Sympos. Pure Math. 56, Part 1, Amer. Math. Soc., 1994, 1-23.

[6] M. Demazure, Invariants symétriques entiers des groupes de Weyl et torsion, Invent. Math. 21 (1973), 287-301.

[7] H. Duan, Multiplicative rule of Schubert classes, Invent. Math. 159 (2005), 407-436.

[8] H. Duan and X. Zhao, The Chow rings of generalized Grassmannians, arXiv:math. AG/0511332.

[9] A. Grothendieck, Torsion homologique et sections rationnelles, in: Anneaux de Chow et applications, Sém. C. Chevalley, 1958, exp. 5, 29 pp., Secrétariat mathématique, Paris.

[10] H. Hiller, Schubert calculus of a Coxeter group, Enseign. Math. (2) 27 (1981), 57-84.

[11] A. Iliev and L. Manivel, The Chow ring of the Cayley planes, Compos. Math. 141 (2005), 146-160.

[12] S. Kaji and M. Nakagawa, The Chow rings of the algebraic groups $\mathrm{E}_{6}$ and $\mathrm{E}_{7}$, arXiv:math.AT/07093702.

[13] R. Marlin, Anneaux de Chow des groupes algébriques $S O(n)$, Spin $(n), G_{2}$ et $F_{4}$, Publ. Math. Orsay 95-7419 (1974).

[14] M. Nakagawa, The integral cohomology ring of $E_{7} / T$, J. Math. Kyoto Univ. 41 (2001), 303-321.

[15] - The integral cohomology ring of $E_{8} / T$, in preparation.

[16] P. Pragacz, Multiplying Schubert classes, in: Topics in Cohomological Studies of Algebraic Varieties, Trends in Math., Birkhäuser, Basel, 2005, 163-174.

[17] - Algebro-geometric applications of Schur $S$ - and Q-polynomials, in: Topics in Invariant Theory, M. P. Malliavin (ed.), Lecture Notes in Math. 1478, Springer, 1991, 130-191.

[18] H. Toda, On the cohomology ring of some homogeneous spaces, J. Math. Kyoto Univ. 15 (1975), 185-199.

[19] H. Toda and T. Watanabe, The integral cohomology ring of $F_{4} / T$ and $E_{6} / T$, ibid. 14 (1974), 257-286.

Department of General Education

Takamatsu National College of Technology

Takamatsu 761-8058, Japan

E-mail: nakagawa@takamatsu-nct.ac.jp

Received 14 September 2007;

in revised form 28 January 2008 